\title{
Corona Virus Disease (COVID-19) and Pregnancy: What Obstetrician Should Know
}

\author{
Jaideep Malhotra ${ }^{1}$, Prabhat Agrawal ${ }^{2}$, Ruchika Garg ${ }^{3}$, Narendra Malhotra ${ }^{4}$, SV Singh $^{5}$ \\ Journal of South Asian Federation of Obstetrics and Gynaecology (2019): 10.5005/jp-journals-10006-1744
}

Pneumonia outbreak of coronavirus disease of 2019 (COVID-19) is caused by the severe acute respiratory syndrome coronavirus 2 (SARS-CoV-2), a novel enveloped RNA betacoronavirus. It infects host respiratory epithelial cells through angiotensin-converting enzyme 2 (ACE2) - a membrane-bound aminopeptidase that functions as its putative receptor. Keeping with the epidemiological observation, majority of COVID-19 infections to date have occurred in men.

COVID-19 is CoV infection identified in December 2019 in Wuhan, a city in Hubei. As on March 23, Europe has become the epicenter of the COVID-19 pandemic, but the infection has spread in more than 150 countries and is a pandemic. This has led governments to adopt rigorous measures to reduce the spread both by lockdown and cancelling most visas. It has detrimental effects on health-care systems and on the whole economy of many countries including the United States.

\section{Symptoms}

The most common symptoms at onset of COVID-19 illness are fever, cough, and fatigue, while other symptoms include sputum production, headache, hemoptysis, diarrhea, dyspnea, and lymphopenia. Clinical features revealed by a chest computed tomography (CT) scan presented as pneumonia, acute respiratory distress syndrome, acute cardiac injury, and incidence of groundglass opacities that lead to death. The symptoms appear after an incubation period of approximately 5.2 days. The period from the onset of COVID-19 symptoms to death ranged from 6 to 41 days with a median of 14 days. ${ }^{1,2}$

Symptoms of COVID-19 and earlier betacoronavirus such as fever, dry cough, dyspnea, lymphopenia, and bilateral ground-glass opacities on chest CT scans are comparable. COVID-19 showed some unique clinical features that include the targeting of the lower airway as evident by upper respiratory tract symptoms like rhinorrhea, sneezing, and sore throat. Some show an infiltrate in the upper lobe of the lung with increasing dyspnea with hypoxemia. Patients infected with COVID-19 can develop gastrointestinal symptoms like diarrhea, but diarrhea is uncommon in MERS-CoV or SARS-CoV. ${ }^{3}$ Fecal and urine samples should be tested to exclude the alternative route of transmission. ${ }^{4}$

\section{Surface Stability of Coronavirus}

SARS-CoV-2 was more stable on plastic and stainless steel than on copper and cardboard, and the viable virus was detected up to 72 hours after application to these surfaces.

\section{Diagnosis}

Thrombocytopenia and an elevation in the hepatic transaminase enzymes (ALT, AST) may be observed.

\author{
${ }^{1,4}$ Rainbow IVF, Agra, Uttar Pradesh, India \\ ${ }^{2}$ Department of Medicine, SN Medical College, Agra, Uttar Pradesh, \\ India; GLA University, Mathura, Uttar Pradesh, India \\ ${ }^{3}$ Department of Gynecology, SN Medical College, Agra, Uttar Pradesh, \\ India
}

${ }^{5}$ Department of Biotechnology, GLA University, Mathura, Uttar Pradesh, India

Corresponding Author: Ruchika Garg, Department of Gynecology, SN Medical College, Agra, Uttar Pradesh, India, Phone: +91 9720004485, e-mail: ruchikagargagra@gmail.com

How to cite this article: Malhotra J, Agrawal P, Garg R, et al. Corona Virus Disease (COVID-19) and Pregnancy: What Obstetrician Should Know. J South Asian Feder Obst Gynae 2019;11(6):337-339.

Source of support: Nil

Conflict of interest: None

Chest X-ray, chest CT scan, and real-time PCR (RT-PCR) or nucleic acid amplification test (NAAT) aid in diagnosis. Confirmation requires nucleic acid testing of respiratory tract samples (e.g., throat swabs), but the clinical diagnosis may be made based on symptoms, exposures, and chest imaging. Specimens for RT-PCR or NAAT should be obtained from the oropharynx and the nasopharynx using a synthetic-tipped applicator with an aluminum shaft (it has sensitivity of $73 \%)^{5,6}$

Intubated patients should have specimens obtained by the bronchoalveolar lavage. The virus also has been recovered from blood and stool, but not yet from urine, amniotic fluid, placenta, cord blood, or breast milk. ${ }^{3}$

The sensitivity, specificity, the positive predictive value (PPV), and the negative predictive value (NPV) of a CT chest in diagnosing COVID-19 in China are 97, 25, 65, and 83\%, respectively (using RT-PCR as a reference)..$^{3,7}$

The teratogenic effects of ionizing radiation on the fetus are inevitable for $\mathrm{CT}$.

Exposure to radiation doses less than $50 \mathrm{mGy}$ is not associated with an increased risk of fetal anomalies or pregnancy loss. The dose of fetal radiation exposure for a routine CT chest is $0.03 \mathrm{mGy}$. Placenta studies have not demonstrated teratogenicity or thyroid dysfunction in the newborn by the intravenous iodinated contrast media (as it crosses the placenta). ${ }^{4}$

\section{TREATMENT}

There is currently no strong evidence that ibuprofen can make coronavirus (COVID-19) worse. But until we have more information, paracetamol can be given to treat the symptoms of coronavirus, unless paracetamol is not suitable to patients.

() The Author(s). 2019 Open Access This article is distributed under the terms of the Creative Commons Attribution 4.0 International License (https://creativecommons. org/licenses/by-nc/4.0/), which permits unrestricted use, distribution, and non-commercial reproduction in any medium, provided you give appropriate credit to the original author(s) and the source, provide a link to the Creative Commons license, and indicate if changes were made. The Creative Commons Public Domain Dedication waiver (http://creativecommons.org/publicdomain/zero/1.0/) applies to the data made available in this article, unless otherwise stated. 


\section{Chloroquine}

It blocks infection by coronavirus by increasing the endosomal $\mathrm{pH}$ required for cell fusion and by interrupting the glycosylation of cellular receptors of SARS-CoV in cell culture.

\section{Antivirals}

\section{Treatment and Management}

Because this condition may be confused with influenza A or B, initial treatment with a drug like oseltamivir $75 \mathrm{mg}$ orally twice daily for 5 days might be considered.

Ribavirin, an antiviral guanosine analog commonly used empirically, is teratogenic: Miscarriage, craniofacial, and limb defects have been attributed to embryos of pregnant mice exposed to doses exceeding $25 \mathrm{mg} / \mathrm{kg}$. Avoid its use in early pregnancy.

Antiviral drugs remdesivir $A$ and chloroquine have been approved by the U.S. Food and Drug Administration for treatment of COVID-19 without completed clinical trials.

The National Institutes of Health is currently conducting a trial of remdesivir. China is promoting favipiravir antiviral (AVIGAN) with promising results. High-dose vitamin $\mathrm{C}$ has also been found to be useful according to a few Chinese reports.

\section{Vaccination}

Currently, there are no approved vaccines for the prevention of COVID-19, although several are under development but will not be available for some time.

\section{COVID-19 in Pregnancy}

Physiological changes in pregnancy encourage rapid progression to respiratory failure in the gravida. The pregnancy bias toward T-helper 2 (Th2) system dominance, which protects the fetus, leaves the mother vulnerable to viral infections, which are more effectively contained by the Th1 system. COVID-19 cannot be considered as an indication for delivery.

The timing and mode of delivery should be individualized according to maternal clinical conditions or obstetric factors. Only limited data have been reported till date. Mothers infected with COVID-19, more than $90 \%$ of whom also had pneumonia, are at increased risks of miscarriage, preterm birth, preeclampsia, cesarean, and their babies are at higher risk of perinatal death and admission to the neonatal intensive care unit (NICU), compared to the general population. There have been no published cases of the clinical evidence of vertical transmission. These data may be updated soon.

Fetal complications of COVID-19 include miscarriage (2\%), intrauterine growth restriction (IUGR; 10\%), and preterm birth (39\%). Fever, with a median temperature of $38.1-39.0^{\circ} \mathrm{C}$, is the prevailing symptom in COVID-19; cohort studies in patients with other infections have not shown increased risks of congenital anomalies from maternal pyrexia in the first trimester but there is a possibility. Childhood inattention disorders are more common, possibly related to hyperthermic injury to fetal neurons. One cannot conclude that the rate of miscarriage and preterm should be attributed solely to the virus/infection, since there are no control groups of uninfected women. Stress in the community might be contributory.

More than $90 \%$ of hospitalized pregnant women affected by $\mathrm{CoV}$ infections present radiological signs of pneumonia, detected at chest X-ray or CT, and the most common symptoms are fever, cough, and lymphopenia. Pregnancies affected by CoV infections have high rates of preterm birth (PTB), before 37 and 34 weeks, and miscarriage when the infection is acquired earlier in pregnancy. Preeclampsia and cesarean delivery are also more common compared to general population. Perinatal mortality is about $10 \%$, while the most common adverse perinatal outcome is fetal distress, with more than half of the newborn admitted in NICU.

According to a retrospective nonrandomized review, it is difficult to draw any convincing evidence on these clinical management strategies. This is very short-term follow-up data and thus infectious that occurred proximate to the delivery. The magnitude of risks of PTB might be overestimated. There was no data on miscarriage for MERS infection occurring during the first trimester. The rate of PTB was $32.1 \%$, all occurring before 34 weeks of gestation. Preeclampsia occurred in $19.1 \%$, while no case of preterm premature rupture of membranes (PPROM) or fetal growth restriction (FGR) was reported in these studies. The rate of perinatal death was $33.2 \%$ including two stillbirths and one neonatal death (4 hours after birth of an extremely preterm infant). ${ }^{4}$

No case of fetal distress, neonatal asphyxia, and admission to NICU was reported. Apgar score was 7 at 5 minutes. There was no evidence of vertical transmission during the follow-up period. Six studies have reported information on COVID-19 infection during pregnancy. There was no data on miscarriage for COVID-19 infection occurring during the first trimester. The rates of PTB for less than 37 and 34 weeks of gestation were 41.1 and 15\%, respectively; PPROM occurred in $18.8 \%$ while the rate of preeclampsia was $13.6 \%$ (1/12-95\% Cl L2-36.0), with no reported cases of FGR. The rate of cesarean delivery was $91 \%$.

The rate of perinatal death was $7 \%(2 / 41-95 \% \mathrm{Cl} 1.4-16.3)$ including one stillbirth and one neonatal death: $43 \%$ of fetuses had fetal distress and $8.7 \%(1 / 10-95 \% \mathrm{Cl} 0.01-31.4)$ of newborns were admitted to NICU.

In a small study conducted on women in their third trimester who were confirmed to be infected with the coronavirus, there was no evidence that there is transmission from the mother to the child. However, all pregnant mothers underwent cesarean sections, so it remains unclear whether transmission can occur during vaginal birth. This is important because pregnant mothers are relatively more susceptible to infection by respiratory pathogens and severe pneumonia.

\section{Breastfeeding}

There is no evidence that the illness can be transmitted through breast milk. If a mother is too ill to breastfeed, she should be encouraged to express milk for the baby, while taking all necessary precautions. A mechanical breast pump should be used to obtain milk and then another family member should feed the baby until the mother tests negative for the virus. The breast pump needs to be cleaned meticulously after each use. Another healthy family or staff member should be present to provide care (e.g., diapering, bathing) and feeding for the newborn; they should use the appropriate personal protective equipment (PPE). For healthy family members, appropriate PPE includes gown, gloves, face mask, and eye protection; use controls like physical barriers (e.g., a curtain between the mother and newborn) and keep the newborn $\geq 6$ feet away from the ill mother.

If there is no one to care for the newborn, a mother who has confirmed COVID-19 should put on a facemask and practice hand hygiene before each feeding or other close contact with the newborn. The face mask should remain in place during contact with 
the newborn. Hand hygiene includes use of the alcohol-based hand sanitizer that contains 60-95\% alcohol before and after all patient contact, contact with the potentially infectious material, and before putting on and upon removal of PPE, including gloves; washing with soap and water for at least 20 seconds is also effective. If hands are visibly soiled, use soap and water before use of the alcohol-based hand sanitizer. Outpatient visits, e.g., routine low-risk antenatal visits for patients having no complaints, gynecological complaints of a chronic nature, and new treatment for fertility care, should be delayed.

\section{Guidelines for Fertility Care as on March 28, 2020}

- Do not initiate new treatment cycles, including ovulation induction, intrauterine insemination (IUI), in vitro fertilization (IVF) (both egg retrievals and frozen embryo transfers [FETs]), and nonurgent egg or embryo freezing.

- Strongly consider canceling all embryo transfers (fresh or frozen),

- Continue caring for people who are "in-cycle" or have urgent needs for stimulation or cryopreservation (egg or embryo freezing).

- Postpone elective surgeries and any nonurgent diagnostic procedures.

- Prioritize telehealth over in-person contact.

- Age and diminished ovarian reserve are time-sensitive; at present, these should not be included in the definition of urgent care.

- Assisted reproductive technology is considered an elective procedure.

- Anyone who is actively pursuing antiretroviral therapy (ART) and meets the diagnostic criteria for the COVID-19 infection should consider freezing all eggs or embryos and wait until they're disease-free to do an embryo transfer.

- Patients who have high likelihood of having COVID-19 (fever and/ or cough, shortness of breath, and either exposure within 6 feet of a confirmed COVID-19 patient and within 14 days of onset of symptoms or a positive COVID-19 test result), including those planning to use oocyte donors, sperm donors, or gestational carriers, should strive to avoid a pregnancy.

- If these patients are undergoing active infertility treatment, we suggest that they consider freezing all oocytes or embryos and avoid an embryo transfer until they are disease-free.

- These recommendations do not necessarily apply when there solely is a suspicion of COVID-19, because symptoms of COVID19 are very similar to other more common forms of respiratory illnesses.

- No new ovulation induction, IUI, fresh IVF, or FET cycles can be started until further notice.

- If currently in a treatment cycle (having started drugs or are being monitored in a natural FET cycle) and patient wishes to continue in a fresh IVF cycle, all embryos should be frozen rather than transferred fresh. Once the situation has settled, then a FET cycle, either natural or medicated, would be undertaken.

\section{Is Sex Possible during this Pandemic?}

There is no evidence that the COVID-19 can be transmitted via either vaginal or anal intercourse. However, virus can be transmitted via saliva. Therefore, the virus can be transmitted by kissing. There is also evidence of the oral-fecal transmission of the COVID-19 and that implies that analingus may represent a risk for infection. Try to keep some physical distance of about 6 feet from other people. That would definitely make sex a challenge. COVID-19 could be transmitted before symptom onset. Masturbation is safe.

\section{N95 Masks: What We do not Know?}

The use of $\mathrm{N} 95$ respirators (also known as FFP2 masks) is recommended by the $\mathrm{CDC}$ for healthcare providers with high-risk exposure to patients with suspected or proven COVID-19 infection. ${ }^{3}$ These are associated with resistance to the airflow, although there are no changes in the fetal heart rate, maternal capillary lactate levels, or oxygen saturations. There is increased carbon dioxide production (9\%) due to labored breathing. ${ }^{4}$

The use of $\mathrm{N} 95$ respirators in pregnant healthcare workers with IUGR is not recommended and it is recommended that they be exempted from frontline duty during the COVID-19 outbreak.

\section{Conclusion}

COVID-19 has mortality of 3-11\%. Acute respiratory distress syndrome, often requiring invasive ventilation, is the clinical epiphenomenon of the viral pneumonia. Data are changing everyday. So, we might have to update it soon.

\section{References}

1. Guan W, Ni Z, Hu Y, et al. Clinical characteristics of coronavirus disease 2019 in China.

2. Engl N, J Med 2020; published online Feb 28. 10.1056/NEJMoa2002032 8. WHO coronavirus disease 2019 (Covid-19) situation report 46, Available at: https://www.who.int/docs/default-source/ coronaviruse/situation-reports/20200306-sitrep-46-covid-19. pdf?sfvrsn=96b04adf_2. Accessed 14 March 2020.

3. Dashraath P, Jing Lin Jeslyn W, Mei Xian Karen L, et al. Coronavirus Disease 2019 (COVID-19) Pandemic.

4. Pregnancy, American Journal of Obstetrics and Gynecology Maternal Fetal Medicine. https://www.thelancet.com 10.1016/S01406736(20)30360-3.

5. Wu Z, McGoogan JM. Characteristics of and Important Lessons From the Coronavirus Disease 2019 (COVID-19) Outbreak in China. Summary of a Report of 72314 Cases From the Chinese Center for Disease Control and Prevention. JAMA 2020;323(13):1239-1242. DOI: 10.1001/jama.2020.2648.

6. Tuite AR, Victoria NG. Estimation of COVID-19 outbreak size in Italy March 19, 2020 Lancet Infectious disease https://www.thelancet. com/journals/laninf/article/PIIS1473-3099(20)30227-9.

7. Centers for Disease Control and Prevention. Coronavirus disease 2019: Cases in US. CDC website. https://www.cdc.gov/coronavirus/2019 ncov/cases-updates/cases-in-us.html. Accessed March 18, 2020. 